\title{
CIT-5: a high-silica zeolite with 14-ring pores
}

\author{
Paul Wagner, ${ }^{a}$ Masahito Yoshikawa, ${ }^{a} \dagger$ Mark Lovallo, ${ }^{b}$ Katsuyuki Tsuji, ${ }^{a}$ Michael Taspatsis ${ }^{b}$ and \\ Mark E. Davis*a \\ a Division of Chemistry and Chemical Engineering, California Institute of Technology, Pasadena, CA 91125, USA \\ ${ }^{b}$ Department of Chemical Engineering, University of Massachusetts, Amherst, MA 01003, USA
}

The synthesis and structure of a new zeolite, CIT-5 (California Institute of Technology Number Five), is described, which possesses one-dimensional pores comprised of $14 \mathrm{~T}$-atoms (tetrahedrally coordinated silicon or aluminium atoms).

Zeolites with pores comprised of larger than $12 \mathrm{~T}$-atoms (extralarge pores) are much in demand ${ }^{1-4}$ and have been so for many years. ${ }^{4-7}$ The reason for this is the desire to perform catalysis/ adsorption on molecules $>8 \AA$ in size.

The first molecular sieve with extra-large pores was VPI-5 that is an aluminophosphate material with 18-ring pores. ${ }^{8}$ Subsequently, other phosphate-based extra-large pore materials have been reported, e.g. cloverite. ${ }^{2}$ All of the phosphate-based molecular sieves lack the desired properties of combined high acidity and thermal/hydrothermal stability and thus limit the practical potential. Recently, the first extra-large pore zeolite, UTD-1, was reported and shown to possess good acidity and thermal/hydrothermal stability. ${ }^{9,10}$ Additionally, there have been numerous disclosures of ordered, aluminosilicate mesoporous materials with pore sizes of $20-100 \AA .11,12$ Because the inorganic portions of the mesoporous materials are not crystalline, they lack the acidity and thermal/hydrothermal stability of high-silica zeolites. ${ }^{13}$

Here, we report a new extra-large pore zeolite denoted CIT-5. CIT-5 is synthesized under hydrothermal conditions. A reaction mixture of composition $0.2 \mathrm{ROH}: 0.1 \mathrm{LiOH}: 0.02 \quad \mathrm{Al}_{2} \mathrm{O}_{3}: 1$ $\mathrm{SiO}_{2}: 40 \mathrm{H}_{2} \mathrm{O}$ is heated to $175^{\circ} \mathrm{C}$ at autogenous pressure for $c a$. $12 \mathrm{~d}$ in order to produce CIT-5. In the absence of aluminium, pure-silica CIT- 5 can be prepared in $5 \mathrm{~d}$. The organic structuredirecting agent (SDA), R, is $N(16)$-methylsparteinium $\mathbf{I}$ and is prepared as previously reported. ${ }^{14}$ In the absence of lithium, I can form pure-silica or borosilicate SSZ-24.13,14 Thus, the key to the successful preparation of CIT-5 is both the SDA I and the inclusion of lithium. Further synthetic details are forthcoming. ${ }^{15}$

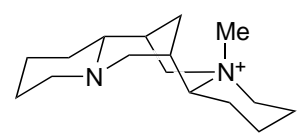

I

Indexation $^{16}$ of the synchrotron powder X-ray diffraction (SPXRD) data from the pure-silica sample of CIT-5 revealed the presence of a small amount of an impurity phase together with the predominant CIT-5 phase. The CIT-5 material indexed in the orthorhombic crystal class with refined lattice constants $a=13.694(2), b=5.0213(5)$, and $c=25.4970(3) \AA$ $\left(U=1753.2 \AA^{3}\right)$. The lattice constants for the impurity hexagonal phase were found to be $a=13.63$ and $c=8.30 \AA$. The unit cell parameters and scanning electron micrographs helpd to identify the impurity phase as SSZ-24 (International Zeolite Association Code AFI). ${ }^{17}$ Systematic absences for the orthorhombic CIT-5 phase indicate body-centering consistent with six possible space groups.

The starting model for Rietveld refinement of CIT-5 was obtained by an iterative process of model building, distance-

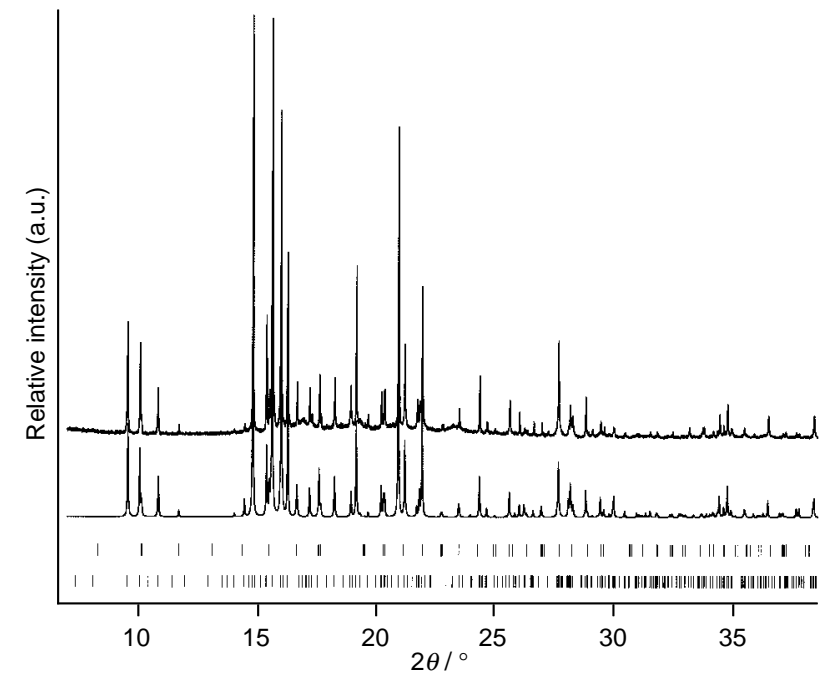

Fig. 1 Experimental (top) and simulated (bottom) powder XRD data of CIT$5, \lambda=1.20028(5) \AA$. The upper tick marks represents peak positions for the impurity phase, SSZ-24, while the bottom set of tick marks represents peak positions for CIT-5.

least squares (DLS) ${ }^{18}$ refinement of the atomic positions for the model, and comparison of the simulated powder X-ray pattern $\left(\right.$ CERIUS $\left.^{19}\right)$ to the experimental pattern. The model with the closest match between the simulated X-ray powder pattern and the experimental XRD was found to have maximal topological symmetry of space group Imma (no. 74, standard setting) (consistent with the systematic absences).

A two phase Rietveld refinement ${ }^{20}$ was carried out using the structural parameters previously reported for the SSZ-24 impurity phase. ${ }^{13}$ The background, scale factor and zero shift were refined, followed by refinement of the lattice parameters, peak shape function parameters and atomic positions for both

Table 1 Crystallographic and refinement data

$\begin{array}{ll}\text { Space group } & \text { Imma (no. 74) } \\ a / \AA & 13.6947(2) \\ b / \AA & 5.0213(5) \\ c / \AA & 25.4970(3) \\ \lambda / \AA & 1.20028(5) \\ T / \mathrm{K} & 298 \\ \text { Profile range/ } & { }^{\circ} \\ \text { Step scan increment } /{ }^{\circ} & 5-42.2 \\ \text { No observation } & 0.005 \\ \text { No. structural parameters } & 7440 \\ \text { No. profile parameters refined } & 55 \\ \text { No. background parameters } & 5 \\ R_{\mathrm{wp}}(\%) & 20 \\ R_{\mathrm{p}}(\%) & 11.43 \\ \text { DW }_{\mathrm{d}}{ }^{a} & 8.75 \\ \text { Reduced }^{2} & 0.040 \\ \end{array}$

a No serial correlation in fit at $90 \%$ confidence for $1.950<\mathrm{DW}_{\mathrm{d}}<$ 2.050 . 


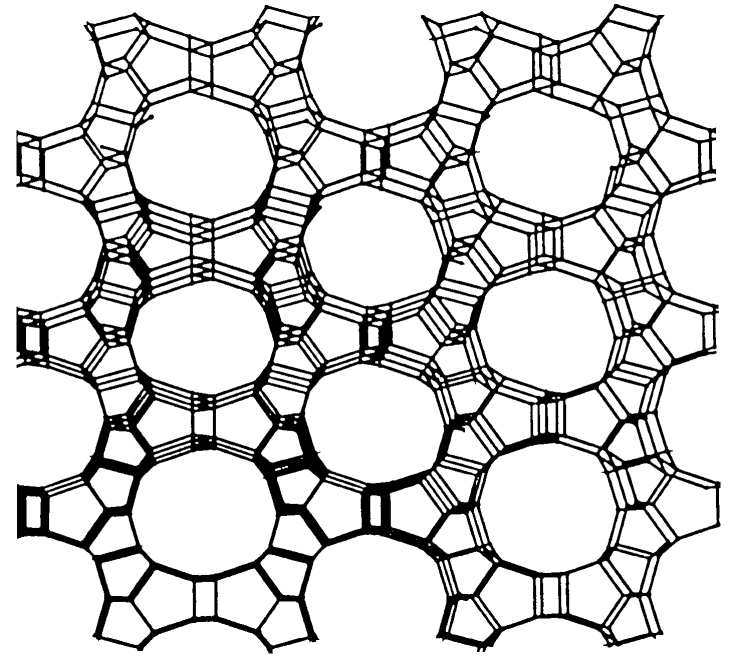

Fig. 2 The [010] projection of CIT-5 showing the 14MR pores

CIT-5 and SSZ-24. Isotropic thermal parameters for the CIT-5 phase were also refined. The phase fraction refinement indicated $c a$. 4\% SSZ-24 impurity. The low values of the residuals $\left(R_{\mathrm{p}}=8.7 \%\right.$ and $\left.\mathrm{R}_{\mathrm{wp}}=11.4 \%\right)$ indicate close agreement between the calculated intensities from the refinement and the experimentally observed intensities (Fig. 1). Further details of the refinement results can be found in Table 1.

The structure of CIT-5 viewed down the $b$-axis is shown in Fig. 2 and is composed of one-dimensional, extra-large pores of nearly circular cross section (center of oxygen to center of oxygen distance $10.0 \times 9.9 \AA$ ) circumscribed by 14 -T-atommembered rings (14MRs). The asymmetric unit contains 5 T-atoms and 8 oxygens resulting in a unit cell content of $\left[\mathrm{Si}_{32} \mathrm{O}_{64}\right]$, a framework density (FD) of $18.3 \mathrm{~T}$-atoms/1000 $\AA^{3}$, and a density of $1.821 \mathrm{~g} \mathrm{~cm}^{-3}$. Table 2 contains the final atomic positional parameters and isotropic temperature factors obtained from the Rietveld refinement.

Comparison of the simulated, experimental and image processed experimental high resolution transmission electron micrographs (HRTEM) of CIT-5 viewed down the pores ([010] projection) are shown in Fig. 3, and confirm the topology of the structure. The primary building unit of CIT-5 is a zigzag ladder of four-membered rings (4MRs) with pendant five-membered rings (5MRs). These units are interconnected through single zigzag chains to form the body centered structure shown in Fig. 2. Electron diffraction patterns of CIT-5 show no signs of streaking indicating that the structure is not faulted or disordered.

Thermogravimetry showed an 11.2 mass $\%$ loss from I by $700{ }^{\circ} \mathrm{C}$ (in air). This amount of organic gives a pore volume of

Table 2 Refined atomic positional parameters in space group Imma (No. 74) and isotropic thermal parameters (eds. in parentheses)

\begin{tabular}{lllll}
\hline Atom & $x$ & $y$ & $z$ & $U_{\text {iso }} / \AA^{2}$ \\
\hline $\mathrm{Si}(1)$ & $0.1160(6)$ & 0.2500 & $0.0317(2)$ & $0.042(4)$ \\
$\mathrm{Si}(2)$ & 0.0000 & 0.7500 & $0.2788(6)$ & $0.082(6)$ \\
$\mathrm{Si}(3)$ & $0.2820(5)$ & 0.7500 & $0.1887(3)$ & $0.050(4)$ \\
$\mathrm{Si}(4)$ & 0.0000 & 0.2500 & $0.2177(4)$ & $0.020(4)$ \\
$\mathrm{Si}(5)$ & $0.1936(6)$ & 0.2500 & $0.1479(4)$ & $0.041(3)$ \\
$\mathrm{O}(6)$ & 0.0000 & 0.2500 & $0.0512(6)$ & $0.049(6)$ \\
$\mathrm{O}(7)$ & $0.0853(9)$ & 0.2500 & $0.1737(4)$ & $0.015(6)$ \\
$\mathrm{O}(8)$ & 0.0000 & $0.5776(31)$ & $0.2309(5)$ & $0.110(9)$ \\
$\mathrm{O}(9)$ & $0.1303(9)$ & 0.0000 & 0.0000 & $0.539(9)$ \\
$\mathrm{O}(10)$ & $0.1896(6)$ & 0.2500 & $0.0909(4)$ & $0.060(4)$ \\
$\mathrm{O}(11)$ & 0.2500 & 0.7500 & 0.2500 & $0.086(11)$ \\
$\mathrm{O}(12)$ & $0.0913(11)$ & 0.7500 & $0.3099(5)$ & $0.047(6)$ \\
$\mathrm{O}(13)$ & $0.2640(7)$ & $0.4474(22)$ & $0.1665(4)$ & $0.070(6)$
\end{tabular}

(a)

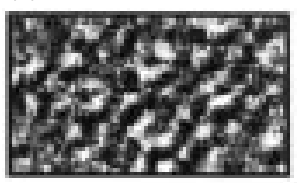

(c)

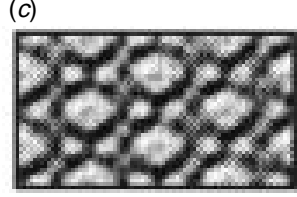

(b)

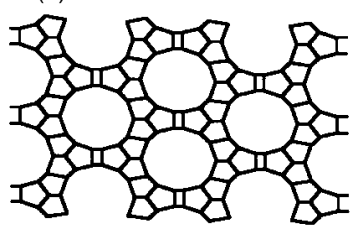

(d)

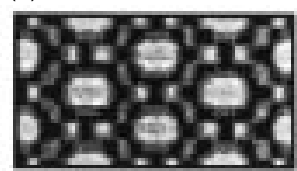

Fig. 3 High-resolution transmission electron micrographs (HRTEM): $(a)$ experimental, $(b)$ [010] projection of CIT-5, $(c)$ processed experimental image and simulated image $(d)$. Samples for HRTEM were prepared by microtomy and images were recorded using a Phillips 430 electron microscope operating at $200 \mathrm{kV}$.

$0.128 \mathrm{ml} \mathrm{g}^{-1}$ and is consistent with the pore volume calculated from the structure $\left(0.135 \mathrm{ml} \mathrm{g}^{-1}\right)$. While CIT-5 contains extralarge 14MR pores, temperature dependent powder XRD studies show that it has very high thermal and hydrothermal stability. The calcined material remains crystalline to over $900{ }^{\circ} \mathrm{C}$, both under pure nitrogen atmosphere and under nitrogen atmosphere containing ca. 23 Torr $\mathrm{H}_{2} \mathrm{O}$.

Financial support for this work was provided by Chevron. We gratefully acknowledged R. Medrud and D. E. Cox for their assistance in collecting the synchrotron powder XRD data. The data were collected at the X7A beamline at the National Synchrotron Light Source at Brookhaven National Laboratory (Upton, NY), that is supported by the Department of Energy, Division of Material Science and Division of Chemical Sciences. P. W. thanks Air Products and Chemicals Inc. for financial support.

\section{Footnotes and References}

* E-mail: mdavis@cheme.caltech.edu

$\dagger$ Current Address: Toray Industries Inc., Nagoya, 455, Japan.

1 M. E. Davis, Chem. Ind. (London), 1992, 4, 137.

2 M. Estermann, L. B. McCusker, Ch. Baerlocher, A. Merrouche and

H. Kessler, Nature, 1991, 352, 320.

3 M. E. Davis, Nature, 1991, 352, 281.

4 M. E. Davis, Nature, 1989, 337, 117.

5 R. M. Barrer and H. Villiger, Z. Kristallogr., 1963, 128, 352.

6 J. V. Smith and W. J. Dytrych, Nature, 1984, 309, 607.

7 G. O. Brunner and W. M. Meier, Nature, 1989, 337, 147.

8 M. E. Davis, C. Saldarriaga, C. Montes, J. Garces and C. Crowder, Nature, 1988, 331, 698.

9 C. C. Freyhardt, M. Tsapatsis, R. F. Lobo, K. J. Balkus, Jr. and M. E. Davis, Nature, 1996, 381, 295.

10 R. F. Lobo, M. Tsapatsis, C. C. Freyhardt, S. Khodabandeh, P. Wagner, C. Y. Chen, K. J. Balkus Jr., S. Zones and M. E. Davis, J. Am. Chem. Soc., 1997, 119, 8474.

11 C. T. Kresge, M. E. Leonowicz, W. J. Roth, J. C. Vartuli and J. S. Beck, Nature, 1992, 359, 710

12 P. T. Tanen and T. J. Pinnavaia, Science, 1995, 267, 865.

13 R. F. Lobo and M. E. Davis, Microporous Mater., 1994, 3, 61.

14 Y. Nakagawa and S. Zones, US Pat., 5271 922, 1993.

15 M. Yoshikawa et al., in preparation.

16 P. E. Werner, L. Erikson and M. Westdahl, J. Appl. Crystallogr., 1985, 18, 367.

17 W. M. Meier and D. H. Olson, Atlas of Zeolite Structure Types, Butterworth, 1987.

18 Ch. Baerlocher, A. Hepp and W. M. Meier, DLS-76: A Fortran Program for the Simulation of Crystal Structures by Geometric Refinement, Institute fur Kristallographie, ETH, Zurich, Switzerland, 1977.

19 CERIUS Version 3.2, Molecular Simulations, Cambridge, UK, 1993.

20 A. C. Larson and R. B. Von Dreele, Los Alamos Laboratory Report, 1987, No. LA-UR-86-748.

Received in Cambridge, UK, 7th July 1997; 7/04774F 